\title{
DIÂMETRO DO FOLÍCULO NO MOMENTO DA INSEMINAÇÃO ARTIFICIAL EM TEMPO FIXO E TAXA DE CONCEPÇÃO EM VACAS NELORE
}

\author{
Antonio De Lisboa Ribeiro Filho ${ }^{1}$, Priscila Assis FerraZ², AleXandra SoARes Rodrigues ${ }^{2}$, \\ THEREZA CRISTINA BORIO DOS SANTOS CALMON BITTENCOURT ${ }^{3}$, MARCUS VINÍCIUS GALVÃo \\ LOIOLA $^{2}$, MARCOS CHALHOUB ${ }^{1}$
}

\author{
${ }^{1}$ Professor Doutor da Universidade Federal da Bahia, Salvador, BA, Brasil. alisboafilho@uol.com.br \\ ${ }^{2}$ Pós-Graduanda da Universidade Federal da Bahia, Salvador, BA, Brasil \\ ${ }^{3}$ Médica Veterinária, Doutora, Universidade Federal da Bahia, Salvador, BA, Brasil
}

\begin{abstract}
RESUMO
Objetivou-se avaliar a influência do diâmetro do maior folículo no momento da IATF (DFOL) sobre a taxa de concepção de fêmeas Nelore submetidas ao protocolo de IATF. Para tanto, foram sincronizadas 348 fêmeas no dia 0 (D0) com um dispositivo intravaginal de progesterona (P4) associado a 2,0mg de benzoato de estradiol por via intramuscular (im). No dia 8 (D8), após a retirada da P4,

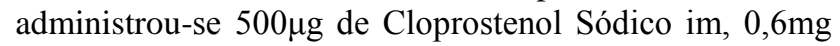
de cipionato de estradiol im e 300UI de gonadotrofina coriônica equina (eCG) im. No dia 10 (D10), antes de cada IATF, mensurou-se o DFOL de todos os animais por ultrassonografia transretal. O diagnóstico de gestação foi

realizado 30 dias após a IATF por ultrassonografia transretal. Os dados foram avaliados pelo pacote estatístico SPSS (versão 19) e as médias do DFOL foram comparadas pelo teste $\mathrm{t}$ de Student. A taxa de concepção geral foi de $57,47 \%$ e a média geral do DFOL foi de $12,43 \pm 2,84 \mathrm{~mm}$. Houve diferença $(\mathrm{P}=0,0001)$ na comparação do DFOL entre as fêmeas gestantes $(13,33 \pm 2,40 \mathrm{~mm})$ e as fêmeas não gestantes $(11,27 \pm 2,75 \mathrm{~mm})$. Os resultados demonstram um efeito positivo do DFOL sobre a fertilidade de vacas Nelore submetidas a um protocolo de sincronização.
\end{abstract}

PALAVRAS-CHAVE: Bovinos; inseminação artificial; sincronização.

\section{FOLLICLE DIAMETER AT FIXED-TIME ARTIFICIAL INSEMINATION AND CONCEPTION RATE IN NELLORE COWS}

\section{ABSTRACT}

The objective of this study was to evaluate the influence of the diameter of the largest follicle at the time of TAI (DFOL) on conception rate of Nellore cows submitted to TAI protocol. Therefore, 348 females were synchronized on day 0 (D0) with an intravaginal progesterone device (P4) associated with $2.0 \mathrm{mg}$ of estradiol benzoate intramuscularly (im). On day 8 (D8), after P4 removal, $500 \mu \mathrm{g}$ Sodium cloprostenol (im), $0.6 \mathrm{mg}$ of estradiol cypionate (im) and 300UI equine chorionic gonadotropin (eCG) (im) were administered. On day 10 (D10), immediately before each TAI, DFOL was measured in all animals by transrectal ultrasonography. Pregnancy diagnosis was performed 30 days after TAI by transrectal ultrasonography. Data were analyzed by SPSS (version 19) and DFOL means were compared by Student's t-test. The overall conception rate was $57.47 \%$ and DFOL overall average was $12.43 \pm 2.84 \mathrm{~mm}$. There were differences $(\mathrm{P}=0.0001)$ in the comparison of DFOL of pregnant $(13.33 \pm 2.40 \mathrm{~mm})$ and nonpregnant $(11.27 \pm 2.75 \mathrm{~mm})$ cows. The results showed a positive effect of DFOL on fertility of cows submitted to synchronization protocol.

KEYWORDS: Artificial insemination; cattle; synchronization. 


\section{INTRODUÇÃO}

A inseminação artificial (IA) é uma importante ferramenta para o melhoramento genético, aumento da eficiência reprodutiva e produtividade dos rebanhos bovinos (BARUSELLI et al., 2006; INFORZATTO et al., 2008). Sendo assim, destaca-se como uma excelente biotecnologia para aperfeiçoar os índices reprodutivos e promover maior retorno econômico para a produção de bovinos (BARUSELLI et al., 2012). Contudo, os índices reprodutivos ainda encontram-se baixos, ocasionados, principalmente, pelas falhas na detecção do estro e anestro pósparto, que afetam o desempenho reprodutivo, especialmente em regiões de clima tropical (BARUSELLI et al., 2004; LARSON et al., 2006).

Neste contexto, diversos protocolos hormonais foram desenvolvidos com a finalidade de controlar o crescimento folicular e induzir a ovulação permitindo, assim, a sincronização do estro e da ovulação, possibilitando o emprego da inseminação artificial em tempo fixo (IATF) sem a necessidade de detecção do estro (BÓ et al., 2003).

Os protocolos mais empregados para sincronização são os que associam estrógeno e progesterona (P4), no início do protocolo hormonal, e prostaglandina $\left(\mathrm{PGF}_{2 \alpha}\right)$, estrógeno e gonadotrofina coriônica equina $(\mathrm{eCG})$, no momento da retirada da fonte de P4 (BÓ et al., 1995; MACMILLAN \& BURKE, 1996). Esses protocolos objetivam sincronizar a emergência da onda folicular, terminar a fase luteínica de forma uniforme e induzir a ovulação sincronizada do folículo dominante ao final do tratamento hormonal (MENEGUETTI et al., 2009). Nesse sentido, diversas pesquisas têm sido conduzidas sobre os fatores associados à resposta ovariana e a fertilidade de vacas de corte submetidas a esses protocolos (MENEGHETTI et al., 2009; PERES et al., 2009). O diâmetro do folículo ovulatório no final do programa de sincronização destaca-se como importante fator de influência nas taxas de concepção e na eficiência reprodutiva dos programas de sincronização para IATF (SÁ FILHO et al., 2009a; SÁ FILHO et al., 2010).

De acordo com SÁ FILHO et al. (2010), o diâmetro do folículo ovulatório está relacionado com maiores concentrações de estradiol, maior probabilidade de ovulação e taxa de concepção.
Assim, a elevada concentração de estradiol préovulatória promovida pelo maior diâmetro do folículo ovulatório pode influenciar na fertilização das fêmeas por promover mudanças no ambiente uterino melhorando o transporte espermático e favorecendo a concepção (SÁ FILHO et al. 2012). Além disso, LONERGAN et al. (2013) relatam que o maior diâmetro do folículo ovulatório também relaciona-se com o maior tamanho do corpo lúteo e, consequentemente, com elevada habilidade em manter a gestação.

O objetivo deste estudo foi avaliar o efeito do diâmetro do maior folículo no momento da IATF sobre a taxa de concepção de vacas Nelore (Bos taurus indicus) submetidas a um protocolo de sincronização do estro e ovulação.

\section{MATERIAL E MÉTODOS}

O experimento foi realizado entre os meses de janeiro a março de 2009 na Fazenda Santa Maria, localizada no município de Maracás, região sudoeste da Bahia. Foram utilizadas 348 fêmeas multíparas da raça Nelore (Bos taurus indicus), com idade média de 5,32 $\pm 0,78$ anos, índice de escore corporal de 2,75 $\pm 0,36$ (IEC - escala de 1 a 5) (HOUGTON et al., 1990) e intervalo pós-parto de $66,2 \pm 0,34$ dias. Os animais foram mantidos em piquete com pastagem predominante de Buffel grass (Cenchrus ciliaris) com suplementação mineral e água ad libitum. A fazenda obedecia ao calendário sanitário oficial e todas as fêmeas foram imunizadas contra doenças da esfera reprodutiva.

Previamente ao início do protocolo de IATF, esses animais foram submetidos a exame clínico-ginecológico e ultrassonografia transretal, utilizando-se um transdutor linear com frequência de 6,0MHz (Pie-Medical, Falco 100, São Paulo, Brasil), sendo considerados aptos a participar do experimento os animais que não apresentassem, no momento da avaliação, anormalidades do trato reprodutivo e sem histórico de aborto.

As fêmeas foram submetidas ao protocolo de sincronização apresentado a seguir. Em um dia aleatório do ciclo estral denominado dia 0 (D0), às 08:00 $\mathrm{h}$, os animais receberam um dispositivo intravaginal de liberação de P4 (Sincrogest ${ }^{\circledR}$, Ouro Fino, São Paulo, Brasil, 1,0g de progesterona) associado a 2,0mg de Benzoato de Estradiol 
(Sincrodiol ${ }^{\circledR}$,Ouro Fino, São Paulo, Brasil, $1 \mathrm{mg} / \mathrm{mL}$ ) por via intramuscular (im). No dia oito (D8), às 08:00 h, retirou-se o dispositivo de $\mathrm{P} 4 \mathrm{e}$ a administração de $500 \mu \mathrm{g}$ de Cloprostenol Sódico (Sincrocio ${ }^{\circledR}$, Ouro Fino, São Paulo, Brasil; 0,25mg/mL) (im); 0,6mg de Cipionato de Estradiol (ECP ${ }^{\circledR}$, Pfizer, São Paulo, Brasil, 2mg/mL) (im) e 300UI de Gonadotrofina Coriônica Equina (Folligon ${ }^{\circledR}$, MSD Saúde Animal, São Paulo, Brasil, 200UI/mL) (im). No dia 10 (D10), às 08:00 h, antes da IATF, todos os animais foram examinados por ultrassonografia transretal com transdutor linear com frequência de $6,0 \mathrm{MHz}$ (PieMedical, Falco 100, São Paulo, Brasil) para mensuração do diâmetro do maior folículo presente no momento da IATF (DFOL). Neste estudo, com a finalidade de evitar efeito do inseminador, as inseminações foram realizadas por um mesmo técnico. Para a realização das inseminações, utilizou-se sêmen criopreservado de um único touro da raça Nelore descongelado a 37 ${ }^{\circ} \mathrm{C}$ por 30 segundos.

O diagnóstico de gestação foi realizado por ultrassonografia transretal 30 dias após as inseminações utilizando-se transdutor linear com frequência de 6,0 MHz (Pie-Medical, Falco 100, São Paulo, Brasil). A presença da vesícula embrionária com um embrião viável (batimento cardíaco) foi considerada diagnóstico de gestação positivo. A taxa de concepção foi calculada dividindo-se o total de vacas gestantes pelo total de vacas inseminadas.

Os dados foram processados pelo Statistical Package for Social Science (SPSS, versão 19) com nível de significância de 5\%. A diferença das médias do DFOL entre os animais gestantes e não gestantes foi comparada pelo teste t de Student. A variável resposta no diagnóstico de gestação foi assumida a apresentar distribuição binomial e foi analisada com base na metodologia de regressão logística. O modelo ajustado pelo procedimento Stepwise para probabilidade de concepção $\left(\mathrm{P}_{\mathrm{i}}\right)$ foi:

$$
P_{i}=\frac{\exp \left(y_{i j}\right)}{1+\exp \left(y_{i j}\right)} \text { em que: }
$$

$\mathrm{Y}_{\mathrm{ij}}=-3,901+0,342$ DFOL. Posteriormente as amostras foram divididas em três categorias a partir da variável DFOL: animais que apresentavam folículos com diâmetro até $11,20 \mathrm{~mm}$ $(\mathrm{n}=113)$, entre 11,20 e $13,60 \mathrm{~mm}(\mathrm{n}=119)$ e acima de $13,60 \mathrm{~mm}(\mathrm{n}=116)$. Para cada animal foi calculada a média de cada grupo pelo teste de Tukey.

\section{RESULTADOS E DISCUSSÃO}

Do total de 348 animais, 200 ficaram gestantes perfazendo uma taxa de concepção de $57,47 \%$. A média geral do DFOL foi de $12,43 \pm 2,84 \mathrm{~mm}$. Na comparação entre o DFOL e a ocorrência ou não de gestação, verificou-se que as vacas que ficaram gestantes apresentaram folículos com diâmetro de $13,33 \pm 2,40 \mathrm{~mm}$, superior $(\mathrm{P}=0,0001)$ ao diâmetro dos folículos das fêmeas não gestantes que alcançaram $11,27 \pm 2,75 \mathrm{~mm}$ (Tabela 1).

Tabela 1 - Média e desvio-padrão (S) do diâmetro do maior folículo no momento da IATF (DFOL) em fêmeas Nelore não gestantes e gestantes

\begin{tabular}{lcc}
\hline Variáveis & Número de animais & $\begin{array}{c}\text { Diâmetro do folículo ovulatório (DFOL) } \\
\text { Média } \pm \mathrm{S}(\mathrm{mm})\end{array}$ \\
\hline Não gestantes & 148 & $11,27 \pm 2,75^{\mathrm{a}}$ \\
Gestantes & 200 & $13,33 \pm 2,40^{\mathrm{b}}$ \\
\hline
\end{tabular}

Valores seguidos de letras distintas na mesma coluna diferem entre si $(\mathrm{P}=0,0001)$.

Corroborando os achados deste estudo, BORSATO et al. (2004) verificaram diferença significativa entre o diâmetro do folículo de novilhas (Bos taurus taurus x Bos taurus indicus) gestantes $(12,31 \pm 2,03 \mathrm{~mm})$ e não gestantes $(6,57 \pm 4,59 \mathrm{~mm})$. PERRY et al. (2007), também observaram que novilhas de corte (Bos taurus taurus) que ovularam folículos menores que $10,7 \mathrm{~mm}$ de diâmetro tiveram menor taxa de concepção comparada com novilhas que ovularam folículos maiores ou iguais a $12,8 \mathrm{~mm}$.

Um achado importante, que pode estar relacionado a esses resultados, encontra-se no fato de fêmeas que ovulam pequenos folículos apresentarem uma reduzida concentração de estradiol no momento da ovulação comparada com fêmeas que ovulam folículos de maior diâmetro ou quando a ovulação 
ocorre de forma espontânea (VASCONCELOS et al., 2001). Dados semelhantes foram observados também por SÁ FILHO et al. (2009b), que verificaram uma proporção linear entre o diâmetro do folículo ovulatório e a concentração de estradiol em vacas Nelore. Da mesma forma, ATKINS et al. (2010) verificaram uma correlação positiva e significativa entre a concentração de estradiol e o diâmetro do folículo ovulatório.

Uma maior concentração fisiológica de estradiol próximo a IA influencia positivamente a fertilização por meio da redução do $\mathrm{pH}$ uterino, modificando o transporte dos espermatozoides e aumentando a longevidade dos mesmos até o momento da ovulação (PERRY \& PERRY, 2008). Por outro lado, a insuficiente secreção de estradiol pelo folículo ovulatório de menor tamanho influencia negativamente o ambiente do útero e do oviduto, comprometendo o transporte dos espermatozoides e a fertilização do oócito (HAWK, 1983). SÁ FILHO et al. (2011) observaram que a presença de folículos de maior diâmetro no momento da remoção do dispositivo de $\mathrm{P} 4$ em um protocolo de sincronização para IATF está associada com uma alta ocorrência de estro, além de uma alta capacidade ovulatória e uma elevada probabilidade de prenhez em vacas zebuínas. Assim, fêmeas que expressaram estro na retirada da fonte de P4 em um protocolo de IATF apresentaram maior taxa de concepção quando comparada àquelas que não expressaram estro, possivelmente por apresentarem diâmetro folicular maior que $14,4 \mathrm{~mm}$ e aumento na produção de estradiol no momento da remoção do dispositivo de P4 (SÁ FILHO et al., 2010).

Além disso, a melhor resposta ovulatória dos folículos de maior diâmetro também pode explicar os resultados encontrados nesta pesquisa, como foi demonstrado por BUTTER et al. (2011), quando estudaram esse fator em novilhas de corte sincronizadas para IATF e observaram que as novilhas que ovularam apresentavam folículos de maior diâmetro em relação as que não ovularam, sugerindo que o sucesso dos protocolos de sincronização depende do crescimento e desenvolvimento do folículo dominante.

Dessa forma, quanto maior o diâmetro do folículo ovulatório maior a capacidade ovulatória desse folículo, o que pode justificar a maior taxa de concepção em fêmeas com maior diâmetro folicular. De acordo com GIMENES et al. (2008), a capacidade ovulatória do folículo em um protocolo hormonal de IATF depende do tamanho folicular quando o indutor da ovulação é aplicado, sendo essa uma das causas da grande variação de resposta aos protocolos hormonais. Esses autores verificaram que a capacidade ovulatória é adquirida quando o folículo dominante atinge entre 7,0 a $8,4 \mathrm{~mm}$ de diâmetro em novilhas Bos taurus indicus e aumenta significativamente quando o folículo alcança $8,5 \mathrm{~mm}$ de diâmetro. DIAS et al. (2009) confirmaram o efeito positivo do diâmetro do maior folículo na probabilidade de concepção em novilhas Nelore sincronizadas para IATF e justificaram tal achado ao aumento dos receptores de LH, melhor resposta à gonadotrofina e maturidade folicular, podendo elevar os índices de fertilidade em novilhas Bos taurus indicus. A expressão gênica de receptores de LH nas células da granulosa em vacas zebuínas ocorre quando o folículo atinge diâmetro maior ou igual a 7,0mm; além disso, o número desses receptores aumenta concomitantemente com o aumento do diâmetro folicular (SIMÕES et al., 2012).

CARVALHO et al. (2008) observaram uma grande proporção de folículos não ovulados em novilhas Bos taurus indicus provavelmente devido à grande proporção de folículos imaturos ao final do protocolo hormonal. Além disso, esses autores relataram que a presença de folículos ovulatórios de menor tamanho contribuiu para falhas e atrasos na ovulação, menor diâmetro do corpo lúteo e, consequentemente, baixa capacidade esteroidogênica. SIQUEIRA et al. (2009) verificaram uma alta correlação entre o volume do tecido luteal e a concentração de $\mathrm{P} 4$, sendo o status funcional do corpo lúteo um indicador de funcionalidade e capacidade esteroidogênica.

A partir do procedimento de regressão logística, foi possível estimar a probabilidade de concepção para cada acréscimo de uma unidade da variável regressora, DFOL (Figura 1), e observou-se que o acréscimo no DFOL levou a um aumento na probabilidade de concepção. Esses resultados são similares aos reportados por BORSATO et al. (2004) que trabalharam com novilhas Bos taurus taurus $\mathrm{x}$ Bos taurus indicus e verificaram um aumento linear entre a taxa de concepção e o diâmetro do folículo ovulatório. Esses autores observaram que animais com folículos ovulatórios entre 7 a $10 \mathrm{~mm}$ obtiveram taxa de concepção de $11,1 \%$, aqueles com folículos de 10 a $13 \mathrm{~mm}$ alcançaram taxa de concepção de $63,8 \%$ e enquanto naqueles com folículos de 13 a $19 \mathrm{~mm}$, foi verificada taxa de concepção de $88,2 \%$.

Neste estudo, os animais foram categorizados em três grupos de acordo com o DFOL e observou-se que no grupo em que as fêmeas apresentaram DFOL acima 13,60mm, a probabilidade de concepção média estimada foi de $78,83 \%$, significativamente superior àquelas que apresentavam diâmetros de até $11,20 \mathrm{~mm}$ e entre 11,20 e $13,60 \mathrm{~mm}$, cujos valores foram respectivamente de $33,78 \%$ e $59,17 \%$. 


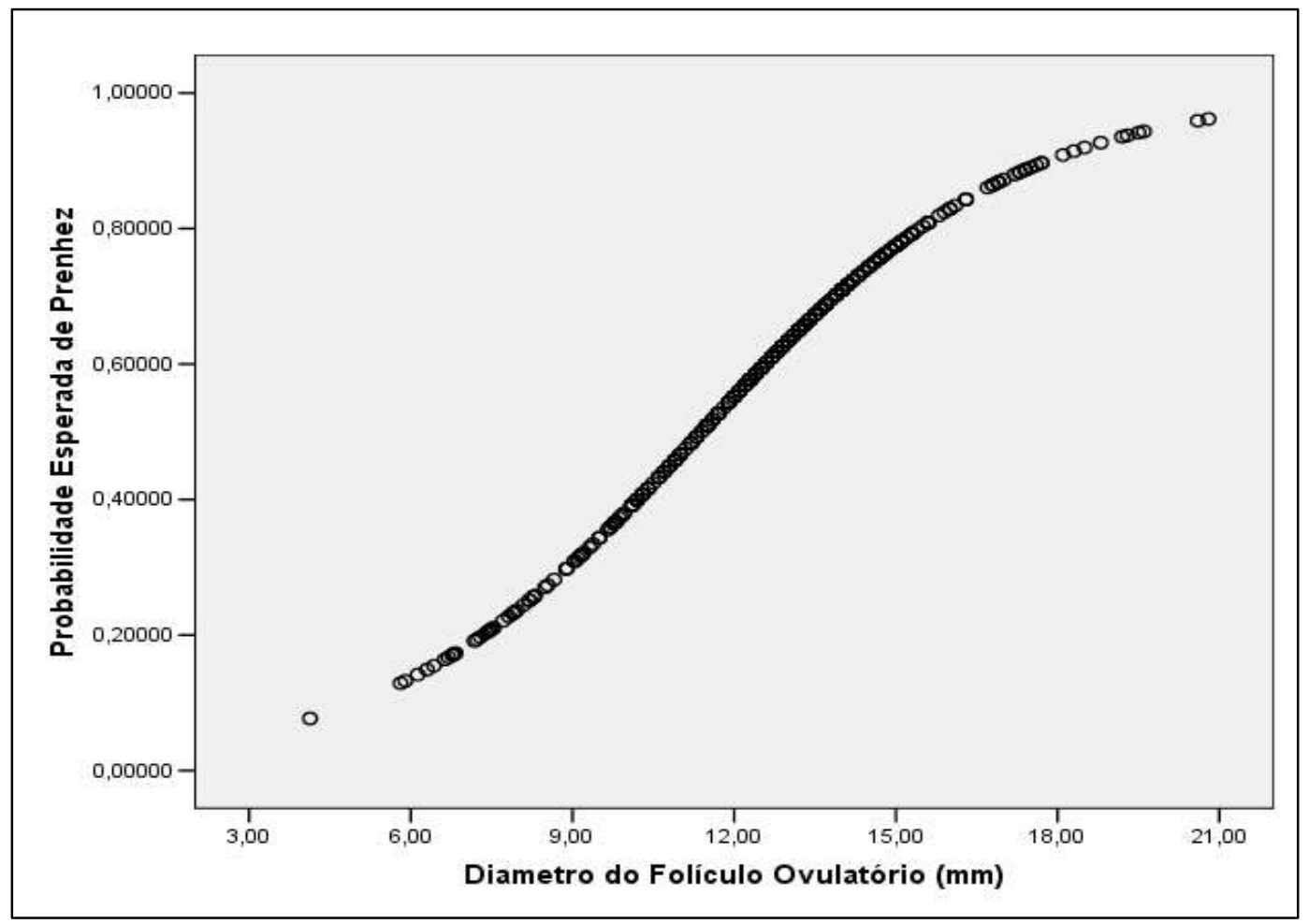

Figura 1 - Probabilidade de concepção em vacas Nelore segundo o diâmetro do maior folículo presente no momento da IATF (DFOL).

SÁ FILHO et al. (2010) também verificaram que existe relação entre o diâmetro do maior folículo e a probabilidade de concepção de fêmeas zebuínas submetidas a protocolos de IATF. Índices satisfatórios de concepção foram alcançados quando o diâmetro do maior folículo no momento da IATF encontrava-se em torno de 13 a $15 \mathrm{~mm}$, assim como foi observado no presente estudo. PERES et al. (2009) e SÁ FILHO et al. (2009a) também observaram relação entre o diâmetro do folículo no momento da IATF e a probabilidade de concepção em vacas Nelore. Da mesma forma, DIAS et al. (2009) relataram um efeito significativo do maior diâmetro folicular no momento da IATF na probabilidade de concepção em novilhas Nelore.

Para PERRY et al. (2005), a ovulação de folículos com diâmetros inferiores a $11 \mathrm{~mm}$ resulta em um aumento na porcentagem de perda embrionária e mortalidade fetal em vacas de corte sincronizadas com o protocolo Ovsynch. De acordo com esses autores, a maior porcentagem de perdas embrionárias pode ser devido a um inadequado desenvolvimento do oócito no momento da ovulação de folículos de menor diâmetro. Além disso, MANN \& LAMMING (2001) relatam que uma diminuição na concentração de $\mathrm{P} 4$ promove uma deficiência no desenvolvimento embrionário, uma baixa capacidade de produzir interferon-tau e, por conseguinte, uma falha na sinalização do embrião para o reconhecimento e estabelecimento da gestação favorecendo, assim, o mecanismo luteolítico.

Dessa maneira, a produção de um embrião viável inclui a ovulação de um oócito competente, uma apropriada produção de $\mathrm{P} 4$ pelo corpo lúteo e um adequado ambiente uterino (PERRY et al., 2007). Assim, uma suficiente secreção de estradiol é necessária para promover um adequado ambiente uterino, secreção pré-ovulatória de LH e mudanças nas células da granulosa para sintetizar progesterona depois da luteinização (PERES et al., 2009).

De acordo com VASCONCELOS et al. (2001), a ovulação de folículos de menor diâmetro pode representar a formação de corpo lúteo de menor volume e, consequentemente, baixa capacidade de produzir $\mathrm{P} 4$ e insuficiente desenvolvimento embrionário, promovendo assim, uma redução na fertilidade.

Segundo PERRY et al. (2005), a maturidade do folículo ovulatório compromete as taxas de concepção em programas de IATF, por afetar o estabelecimento e a manutenção da prenhez em vacas de corte. Entretanto, diversos estudos associados com os resultados do presente experimento têm sugerido que o tamanho do folículo no momento da IATF é um relevante indicador de maturidade folicular e melhor fertilidade, uma vez 
que se observa uma redução da taxa de concepção associada com a ovulação de folículos de menor diâmetro e provavelmente imaturos (PERRY et al., 2007; ATKINS et al., 2010). Além disso, SÁ FILHO et al. (2011) descreveram que a mensuração do diâmetro do folículo ovulatório no momento da IATF é uma importante estratégia para predizer a alta fertilidade em fêmeas zebuínas submetidas a um protocolo de IATF e permitir o direcionamento dos acasalamentos, por exemplo, quando da utilização de sêmen sexado ou de alto valor econômico.

\section{CONCLUSÕES}

A presença de um folículo de maior diâmetro no momento da inseminação é um indicador de melhor resposta ovariana e taxa de concepção de fêmeas Bos taurus indicus submetidas a programas de IATF. Dessa forma, alternativas devem ser adotadas com o intuito de aumentar o diâmetro do folículo no momento da IATF, incrementando a eficiência dos protocolos de sincronização e proporcionando uma melhor relação custo-benefício para o produtor que adota esta biotecnologia.

\section{REFERÊNCIAS}

ATKINS, J. A.; SMITH, M. F.; WELLS, K. J.; GEARY, T. W. Factors affecting preovulatory follicle diameter and ovulation rate after gonadotrophin-releasing hormone in postpartum beef cows. Part I: cycling cows. Journal Animal Science, v.88, p. 2300-2310, 2010.

BARUSELLI, P. S.; SALES, J. N. S.; SALA, R. V.; VIEIRA, L. M.; SÁ FILHO, M. F. History, evolution and perspectives of timed artificial insemination programs in Brazil. Animal Reproduction. v. 9, p. 139-152, 2012.

BARUSELLI, P. S.; REIS, E. L.; MARQUES, M. O.; NASSER, L. F.; BÓ, G. A. The use of hormonal treatments to improve reproductive performance of anestrous beef cattle in tropical climates. Animal Reproduction Science, v. 82-83, p. 479-486, 2004.

BARUSELLI, P. S.; MARQUES, M. O.; CARVALHO, N. A. T.; MADUREIRA, E. H.; CAMPOS FILHO, E. P. Efeito de diferentes protocolos de inseminação artificial em tempo fixo na deficiência reprodutiva de vacas de corte lactantes. Revista Brasileira de Reprodução Animal, v. 26, n. 3, p. 219-220, 2002.

BÓ, G. A.; BARUSELLI, P. S.; MARTINEZ, M. F. Pattern and manipulation of follicular development in Bos indicus cattle. Animal Reproduction Science, v. 78, p.307-326, 2003.

BÓ, G. A.; ADAMS, G.P; PIERSON, R. A.; MAPLETOFT, R. J. Exogenous control of follicular wave emergence in cattle. Theriogenology, v. 43, p. 31-40, 1995.
BORSATO, E. A.; LUDWIG JR., H. E.; RUBIN, K. C. P.; SAUT, J. P. E.; BARREIROS, T. R. R.; SENEDA, M. M. Relação entre o tamanho do folículo ovulatório e taxa de concepção em novilhas Bos taurus x Bos indicus submetidas a inseminação artificial em tempo fixo. Revista Brasileira de Reprodução Animal, v. 28, n. 3, p. 137-142, 2004.

BUTTER, S. A. A.; PHILLIPS, N. J.; BOE-HANSEN, G. B.; BO, G. A.; BURNS, B. M.; DAWSON, K.; MCGOWAN, M. R. Ovarian responses in Bos indicus heifers treated to synchronise ovulation with intravaginal progesterone releasing devices, oestradiol benzoate, prostaglandin F2 $\alpha$ and equine chorionic gonadotrophin. Animal Reproduction Science, v. 129, p. 118-126, 2011.

CARVALHO, J. B. P.; CARVALHO, N. A. T.; REIS, E. L.; NICHI, M.; SOUZA, A. H.; BARUSELLI, P. S. Effect of early luteolysis in progesterone-based timed AI protocols in Bos indicus, Bos indicus x Bos taurus and Bos taurus heifers. Theriogenology, v. 69, p. 167-175, 2008.

DIAS, C. C.; WECHSLER, F. S.; DAY, M. L.; VASCONCELOS, J. L. M. Progesterone concentrations, exogenous equine chorionic gonadotropin, and timing of prostaglandin F2 $\alpha$ treatment affect fertility in postpuberal Nelore heifers. Theriogenology, v. 72, p. 378-385, 2009.

HAWK, H. W. Sperm survival and transport in the female reproductive tract. Journal of Dairy Science, v. 77, p.2738-2744, 1983.

HOUGHTON, P.L.; LEMENAGER, R.P.; MOSS, G.E.; HENDRIX, K. S. Prediction of postpartum beef cow body composition using weight to height ratio and visual body condition score. Journal of Animal Science, v.68, p.1428-1437, 1990.

INFORZATTO, G. R.; SANTOS, W. R. M.; CLIMENI, B. S. O.; DELLALIBERA, F. L.; FILADELPHO, A. L. Emprego da IATF (Inseminação Artificial em Tempo Fixo) como alternativa na reprodução da pecuária de corte. Revista Científica Eletrônica de Medicina Veterinária, v. 11, p. 1-8, 2008.

GIMENES, L. U; SÁ FILHO, M. F.; CARVALHO, N. A. T.; TORRES-JUNIOR, J. R. S.; SOUZA, A. H.; MADUREIRA, E. H.; TRINCA, L. A.; SARTORELLI, E. S.; BARROS, C. M.; CARVALHO, J. B. P.; MAPLETOFT, R. J.; BARUSELLI, P. S. Follicle deviation and ovulatory capacity in Bos indicus heifers. Theriogenology, v. 69, p. 852-858, 2008.

LAMB, G. C.; STEVENSON, J. S.; KESLER, D. J.; GARVERICK, H. A.; BROWN, D. R.; SALFEN, B. E. Inclusion of an intravaginal progesterone insert plus $\mathrm{GnRH}$ and prostaglandin F2 $\alpha$ for ovulation control in postpartum suckled beef cows. Journal Animal Science, v. 79, p. 2253-2259, 2001.

LARSON, J. E.; LAMB, G. C.; STEVENSON, J. S.; JOHNSON, S. K.; DAY, M. L.; GEARY, T. W.; KESLER, D. J.; DEJARNETTE, J. M.; SCHRICK, F. N.; DICOSTANZO A.; ARSENEAU, J. D. Synchronization 
of estrus in suckled beef cows for detected estrus and artificial insemination and timed artificial insemination using gonadotropin-releasing hormone, prostaglandin F $2 \alpha$, and progesterone. Journal of Animal Science, v. 84, p.332-342, 2006.

LONERGAN, P.; O`HARA, L.; FORDE, N. Papel da progesterona do diestro na função endometrial e desenvolvimento do concepto em bovinos. Animal Reproduction, v.10, n. 3, p. 119-123, 2013.

MACMILLAN, K. L.; BURKE, C. R. Effects of oestrous cycle control on reproductive efficiency. Animal Reproduction Science, v. 42, p. 307-320, 1996.

MENEGHETTI, M.; SÁ FILHO, O. G.; PERES, R. F. G.; LAMB, G. C.; VASCONCELOS, J. L. M. Fixed-time artificial insemination with estradiol and progesterone for Bos indicus cows I: Basis for development of protocols. Theriogenology, v. 72, p. 179-189, 2009.

MANN, G. E.; LAMMING, G. E.; Relationship between maternal endocrine environment, early embryo development and inhibition of the luteolytic mechanism in cows. Reproduction, v. 121, p. 175-180, 2001.

PERES, R. F. G.; CLARO JUNIOR, I.; SÁ FILHO, O. G.; NOGUEIRA, G. P.; VASCONCELOS, J. L. M. Strategies to improve fertility in Bos indicus postpubertal heifers and nonlactating cows submitted to fixed-time artificial insemination. Theriogenology, v. 72, p. 681-689, 2009.

PERRY, G. A.; PERRY, B. L. Effect of preovulatory concentrations of estradiol and initiation of standing estrus on uterine $\mathrm{pH}$ in beef cows. Domestic Animal Endocrinology, v. 34, p. 333-338, 2008.

PERRY, G. A.; SMITH, M. F.; ROBERTS, A. J.; MACNEIL, M. D.; GREARY, T. W. Relationship between size of the ovulatory follicle and pregnancy success in beef heifers. Journal Animal Science, v. 85, p. 684-689, 2007.

PERRY, G. A.; SMITH, M. F.; LUCY, M. C.; GREEN, J. A.; PARKS, T. E.; MACNEIL, M. D.; ANDREW, J. R.; THOMAS, W. G. Relationship between follicle size at insemination and pregnancy success. The National Academy of Sciences of the USA, v. 102, p. 5268-5273, 2005.

SÁ FILHO, M. F.; SANTOS, J. E. P.; FERREIRA, R. M.; SALES, J, N. S.; BARUSELLI, P. S. Importance of estrus on pregnancy submitted to estradiol/progesterone- based timed insemination protocols. Theriogenology, v. 76, p.455-463, 2011.

SÁ FILHO, M. F.; CRESPILHO, A. M.; SANTOS, J. E. P.; PERRY, G. A.; BARUSELLI, P. S. Ovarian follicle diameter at timed insemination and estrous response influence likelihood of ovulation and pregnancy after estrous synchronization with progesterone or progestinbased protocols in suckled Bos indicus cows. Animal Reproduction Science, v. 120, p. 23-30, 2010.

SÁ FILHO, O. G.; MENEGHETTI, M.; PERES, R. F. G.; LAMB, G. C.; VASCONCELOS, J. L. M. Fixed-time artificial insemination with estradiol and progesterone for Bos indicus cows II: Strategies and factors affecting fertility. Theriogenology, v. 72, p. 210-218, 2009a.

SÁ FILHO, O. G.; THATCHER, W. W.; VASCONCELOS, J. L. M. Effect of progesterone and/or estradiol treatments prior to induction of ovualation on subsequent luteal lifespan in anestrous Nelore cows. Animal Reproduction Science, v. 112, p. 95-106, 2009 b.

SANTOS, J. E. P.; THATCHER, W.W.; CHEBEL, R. C.; CERRI, R. L. A.; GALVÃO, K. N; The effect of embryonic death rates in cattle on the efficacy of estrus synchronization programs. Animal Reproduction Science, v. 82-83, p. 513-535, 2004.

SIMÕES, R. A. L.; SATRAPA, R. A.; ROSA, F. S.; PIAGENTINI, M.; CASTILHO, A. C. S.; ERENO, R. L.; TRINCA, L. A.; NOGUEIRA, M. F. G.; BURATINI JR, J.; BARROS, C. M. Ovulation rate and its relationship with follicle diameter and gene expression of the $\mathrm{LH}$ receptor (LHR) in Nelore cows. Theriogenology, v. 77, p.139-147, 2012.

SIQUEIRA, L. G.; TORRES, C. A. A.; AMORIN, L. S.; SOUZA, E. D.; CAMARGO, L. S. A.; FERNANDES, C. A. C.; VIANA, J. H. M. Interrelationships among morphology, echtexture, and function of the bovine corpus luteum during the estrous cycle. Animal Reproduction Science. v. 115, p. 18-28, 2009.

VASCONCELOS, J. L. M.; SARTORI, R.; OLIVEIRA, H. N.; GUINTHER, J. G.; WILTBANK, M. C. Reduction in size of the ovulatory follicle reduces subsequent luteal size and pregnancy rate. Theriogenology, v. 56, p. 307314, 2001. 